[Article]

\title{
乙烯基噻吩共轭螺噁嗪化合物的密度泛函理论研究
}

\author{
孙海涛 ${ }^{1}$ 田晓慧 ${ }^{1, *}$ 元以中 ${ }^{1} \quad$ 孙金煌 $^{1} \quad$ 孙真荣 $^{2}$ 卓小玲 ${ }^{1}$ \\ ('华东理工大学材料科学与工程学院, 超细材料制备与应用教育部重点实验室, 上海 200237; \\ 2华东师范大学, 精密光谱科学与技术国家重点实验室, 上海 200062)
}

\begin{abstract}
摘要: 采用密度泛函理论(DFT)方法, 在 B3LYP/6-31G* 水平上对乙烯基噻吩共轭螺噁嗪化合物 SO-SO3 的 几何构型、电子结构、前线分子轨道等进行了理论研究, 计算结果表明: SO-SO3 的开环过程会使得开环体的 左右两个部分键长均等化, 导致共轭体系变大, 能隙明显减小; 乙烯基噻吩基团共轭接入螺噁溙母体后, 导致体 系的共轭作用变大, 在激发态下电子流动增强, 形成由乙烯基噻吩向荟并噁嗪的有效电荷转移与能量转移; 结 合前线分子轨道成分分析乙烯基噻吩单元在最高占据分子轨道(HOMO)和最低未占据分子轨道(LUMO)中的 轨道贡献率明显增加. 含时密度泛函理论(TD-DFT)计算的电子吸收光谱结果显示: 当接入的乙烯基噻吩单元 达到 2-3 个时, 影响 $\mathrm{SO} 2$ 和 $\mathrm{SO} 3$ 开环的最低能量激发态变为第一激发单重态 $S_{1}$, 并且均源自电子从 HOMO 至 LUMO 的跃迁且为 $\pi-\pi ゙$ 跃迁; 其最大吸收波长 $\lambda_{\text {max }}$ 达到 466-540 nm, 且红移十分明显, 其对应开环体 O-SO2 与 O-SO3 的 $\lambda_{\text {max }}$ 达到 605 和 $647 \mathrm{~nm}$.
\end{abstract}

关键词: 乙烯基噻吩; 螺噁嗪; 密度泛函理论; 前线分子轨道; 电子吸收光谱

中图分类号: 0641

\section{Density Functional Theory Study on Vinyl Thiophene Group Conjugated Spirooxazines}

\author{
SUN Hai-Tao ${ }^{1} \quad$ TIAN Xiao-Hui ${ }^{1, *} \quad$ YUAN Yi-Zhong ${ }^{1} \quad$ SUN Jin-Yu ${ }^{1}$ \\ SUN Zhen-Rong ${ }^{2} \quad$ ZHUO Xiao-Ling ${ }^{1}$ \\ ('Key Laboratory for Ultrafine Materials of Ministry of Education, School of Materials Science and Engineering, East China \\ University of Science and Technology, Shanghai 200237, P. R. China; ${ }^{2}$ State Key Laboratory of Precision Spectroscopy, \\ East China Normal University, Shanghai 200062, P. R. China)
}

\begin{abstract}
We carried out a theoretical study on the geometries, electronic structures, and frontier molecular orbitals of vinyl thiophene group conjugated spirooxazines (SO-SO3) using density functional theory (DFT) at the B3LYP/6-31G* level. The calculated results show that the equalization of bond lengths at the left and right parts of the open-forms occurred during the ring-opening process. A large conjugated system was formed and this significantly narrowed the energy gap. The conjugated system became larger and its electrons flowed easily because of the introduction of different lengths of vinyl thiophene conjugation moieties into the spirooxazine molecule. The electrons and energy efficiently transferred from the vinyl thiophene to naphthoxazine. The orbital contribution rate of the vinyl thiophene group in the highest occupied molecular orbital (HOMO) and the lowest unoccupied molecular orbital (LUMO) increased obviously. Time-dependent DFT (TD-DFT) calculations showed that as the conjugated vinyl thiophene unit reached 2-3 the first singlet excited state of $\mathrm{SO} 2$ and $\mathrm{SO} 3$ resulted from the electron transition from the HOMO to the LUMO, which were also assigned to the $\pi-\pi \pi^{*}$ transition. Meanwhile, $\lambda_{\max }$
\end{abstract}

Received: April 21, 2011; Revised: May 18, 2011; Published on Web: July 1, 2011.

"Corresponding author. Email: tianxh@263.net; Tel: +86-21-64252167.

The project was supported by the National High Technology Research and Development Program of China (0099AA03500), Shanghai Leading Academic Discipline Project (B502) and Shanghai Key Laboratory Project, China (08DZ2230500, 09JC1404300).

国家高技术研究发展计划(0099AA03500), 上海市重点学科(B502)和重点实验室(08DZ2230500, 09JC1404300)资助项目

(C) Editorial office of Acta Physico-Chimica Sinica 
was between 466 and $540 \mathrm{~nm}$ with an obvious red-shift while the $\lambda_{\max }$ of O-SO2 and O-SO3 reached 605 and $647 \mathrm{~nm}$, respectively.

Key Words: Vinyl thiophene; Spirooxazine;

Density functional theory; Frontier molecular orbital;

Electronic absorption spectrum

\section{1 引言}

螺噁嗪化合物的光致变色现象首次被 Fox $^{1}$ 报 道, 他合成了第一个螺噁嗪化合物-1,3,-二氢-1,3,3三甲基 [2H]-吲哚螺-2,3'-[3H]荟并 [2,1-b][1,4]婜嗪 (NISO), 后来经证实此类化合物的光化学性质较偶 氮类, 螺吡喃类具有更高的光稳定性和抗疲劳性, 有潜在的应用前景, 因而近年来得到了广泛深入的 研究. ${ }^{2-4}$ 与此同时, 噻吩类化合物作为共轭电致变色 材料的典型代表以其良好的稳定性、可加工性, 独 特的半导体特性和多样的可调节性质同样引起了 人们很大的研究兴趣. ${ }^{2}$ 因此, 将含噻吩单元通过共 轭的方式引入到螺噁嗪母体中制成具有光致变色 和光电转化的新型双功能材料, 在拓展了两类材料 应用的基础上希望能获得新的光学性质, 并且在数 据存储、显示材料、光电材料、生物分子活性的光调 控、光计算以及防伪鉴伪方面具有潜在的应用前 景. 与传统的大量论文和专利报道的吲哚啉萗并螺 噁嗪相比, 对于噻吩共轭螺噁嗪类化合物的报道很 少, 理论研究也相对较少. 随着密度泛函理论的发 展与成熟, 将其应用于许多有机化合物的理论计算 已成为当前一个非常活跃的研究领域. ${ }^{5-7}$ 本文通过 改变乙烯基噻吩共轭基团连接的长度设计了一系 列新型的乙烯基噻吩共轭螺噁嗪衍生物 $\mathrm{SO}-\mathrm{SO} 3$ (图 1), 并通过密度泛函理论, 研究了这类化合物的 结构和性能的关系、电子结构、前线分子轨道以及 吸收光谱性质等, 为进一步设计和合成具有可见光 致变色及光电转化的新型双功能的材料提供比较 可靠的方向性引导和有力的理论依据.

\section{2 计算方法}

在量子化学计算中, 要得到可靠的结果, 计算 模型和基组的合理选择是必要的. ${ }^{8,9}$ 考虑到计算资 源和计算精度等因素, 本文采用密度泛函 DFT/ $\mathrm{B}^{2} \mathrm{LYP}^{10(\mathrm{a}, \mathrm{b})}$ 方法, 以 6-31G* 基组依次对乙烯基噻吩 共轭螺噁嗪衍生物 $\mathrm{SO}-\mathrm{SO} 3$ 及其相应的开环体的 几何构型进行全优化, 分析了分子的结构特点及相 互关系, 计算得到了最高占据轨道(HOMO)、最低空
轨道(LUMO)及能隙. 采用自然键轨道(NBO)分析获 得原子上自然电荷分布, 通过组态相互作用(CIS)计 算得到其基态和第一单激发态的电子结构. 在优化 得到的基态几何构型的基础上, 采用含时密度泛函 $(\mathrm{TD}-\mathrm{DFT})^{10(\mathrm{c}, \mathrm{d})}$ 方法研究了其电子吸收光谱的性质. 所有计算都利用 Gaussian 09 程序 ${ }^{11}$ 并在 Pentittm IV 微机上完成, 计算的收玫精度均采用程序内定值.

\section{3 结果与讨论}

\section{1 几何构型的优化}

如图 1 所示, 吲哚啉螺噁嗪化合物的分子结构 可分为两部分, 左边是吲哚环部分, 右边是菜并噁 嗪部分, 它们以 $s p^{3}$ 杂化的螺碳原子连接, 这两部分 基本上相互垂直, 在紫外光的激发下, SO (spirooxazine) 分子中的螺 $\mathrm{C}-\mathrm{O}$ 键发生异裂, 引起分子的结 构以及电子的组态发生异构化和重排, 通过螺碳原 子连接的两个环系由正交变为共平面, 螺碳原子由 $s p^{3}$ 杂化变为 $s p^{2}$ 杂化, 同时形成了一个大的共轭 体系. 在可见光或热的作用下, 开环体发生关环 反应返回到 $\mathrm{SO}$, 构成了一个典型的光致变色体系. ${ }^{2}$ Schneider ${ }^{12(a)}$ 对螺噁嗪的开环过程进行了研究, 经过 CARS 方法测定和 PPP 方法计算结果证实是各种异 构体的混合物并且至少有四种异构体是可以相对 稳定存在的, 其中最为稳定的是反-反-顺(TTC)构 型. ${ }^{2(b)}$ 为方便讨论及节省计算机时, 文中仅以 TTC 构型作为开环体的代表进行理论计算.

结合图 1 和表 $1^{13}$ 给出了 $\mathrm{SO}-\mathrm{SO} 3$ 及其开环体 $(\mathrm{O}-\mathrm{SO})$ 分子在 B3LYP/6-31G* 水平下优化的部分键 长参数以及文献值, 并且优化得到的键长与文献值 的误差仅为 $1 \%$ 左右, 证实了计算所采用的泛函和 基组对于本体系研究的可靠性. 由表 1 可见, $\mathrm{SO}-\mathrm{SO} 3$ 在变为开环体的过程中, 其化学键 1-6 的 键长发生了明显的变化, 整体趋势为长键变短, 短 键变长, 表明形成的开环体的左右两个部分具有键 长均等化的趋势, 键 1-6 键长的平均值由 $0.1400 \mathrm{~nm}$ 减少至 $0.1361 \mathrm{~nm}$, 说明了开环形成了明显的离域化 结构. 此外, 螺 $\mathrm{C}-\mathrm{O}$ 键(键 7)的键长随着连接的乙烯 


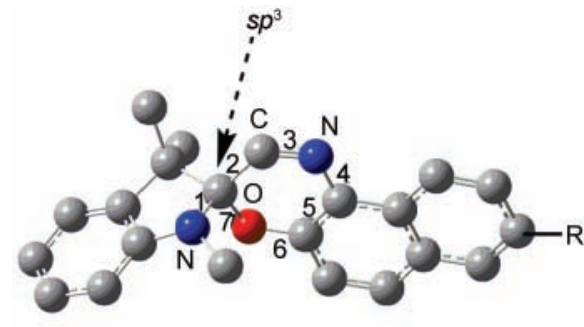

closed-SO

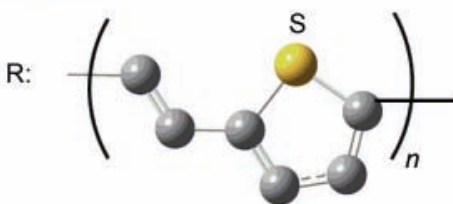

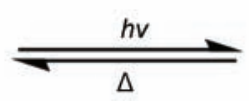

$n=0, \mathrm{SO} ; \quad n=2, \mathrm{SO}$;

图 1 SO-SO3 分子结构模型及示意图

Fig.1 Molecular structural model and sketch map of SO-SO3 $\mathrm{H}$ atoms are omitted for clarity.

基噻吩单元的增加而具有略微增大的趋势, 这使得 在反应过程中键的断裂需要的能量更低, 因此这将 有利于在紫外辐射甚至可见光照射下螺 $\mathrm{C}-\mathrm{O}$ 键的 断裂和开环体的形成. 表 1 还给出了乙烯基噻吩基 团的碳碳单双键交替键长差值(BLA)值, ${ }^{14}$ 所谓 BLA 值就是指对于含有 $\mathrm{CC}$ 单双键交替的化合物或基团 的 $\mathrm{C}-\mathrm{C}$ 键长的平均值和 $\mathrm{C}=\mathrm{C}$ 键长的平均值之差. 由表 1 可见, $\mathrm{SO} 1-\mathrm{SO} 3$ 和对应开环体的乙烯基噻吩 基团的 BLA 值逐渐减小, 表明其共轭体系的扩大; 其中开环体的乙烯基噻吩基团的 BLA 值比相应闭 环体的略小, 表明开环过程中电子云流动增强可以 使螺噁嗪萗环部分与乙烯基噻吩基团共轭作用变 大但是影响不大. 此外计算所得 $\mathrm{SO}-\mathrm{SO} 3$ 的偶极矩 由 $2.7819 \mathrm{C} \cdot \mathrm{m}$ 增加到 $4.8367 \mathrm{C} \cdot \mathrm{m}$, 说明乙烯基噻吩 的引入使得基态分子的极性增大.

\section{2 电子结构}

采用 $\mathrm{NBO}$ 分析获得部分原子和基团上自然电 荷分布. 通过计算发现, 在接入不同长度的乙烯基 噻吩单元后吲哚 $\mathrm{N}$ 原子上的电荷 $(-0.470 e$ ) 并不发 生明显变化, 但从化合物 $\mathrm{SO}$ 到 $\mathrm{SO} 3$ 其噁溙 $\mathrm{O}$ 原子 上的电荷由 $-0.538 e$ 减小到 $-0.536 e$, 说明在荟环上 接入乙烯基噻吩基团后不影响吲哚环部分的电荷 分布, 但是噁嗪 $\mathrm{O}$ 上的电荷密度有减少的趋势, 这 样的电荷变化显然会破坏化合物的稳定性, 使得螺 $\mathrm{C}-\mathrm{O}$ 键的断裂容易, 增大开环速率. 同样比较 $\mathrm{SO}$ 和 SO1 的电荷密度剖面图也可以看出接入乙烯基 噻吩基团后荎并啞嗪环的剖面线条更加均匀, 电荷 密度分布更均匀, 说明共轭效应作用明显增强.

此外, 分别计算了 $\mathrm{SO}-\mathrm{SO} 3$ 的吲哚环(含螺 $\mathrm{C}$ 原 子)、荎并噁嗪、乙烯基噻吩单元三个部分的基态和 第一激发单重态(开环前)的电荷分布(见图 2). 正号 表示有电子流入; 负号表示有电子流出. 由图可见,

表 1 闭环体 SO-SO3 及相应的开环体(O-SO)分子在 B3LYP/6-31G* 水平下优化的部分键长 $(l)$ 和乙烯基噻吩基团的 碳碳单双键交替键长差值 (BLA) (nm)

Table 1 Selected B3LYP/6-31G* skeletal bond lengths $(l)$ for SO-SO3 and the corresponding open-forms $(\mathrm{O}-\mathrm{SO})$ and the mean single-double $\mathrm{CC}$ bond length alternation (BLA) values for vinyl thiophene group

\begin{tabular}{|c|c|c|c|c|c|c|c|c|}
\hline \multirow{2}{*}{ Bond } & \multicolumn{8}{|c|}{$l / \mathrm{nm}$} \\
\hline & $\mathrm{SO}\left(\operatorname{expt}^{13}\right)$ & $\mathrm{O}-\mathrm{SO}$ & SO1 & O-SO1 & $\mathrm{SO} 2$ & $\mathrm{O}-\mathrm{SO} 2$ & $\mathrm{SO} 3$ & $\mathrm{O}-\mathrm{SO} 3$ \\
\hline 1 & $0.1453(0.1435)$ & 0.1371 & 0.1453 & 0.1370 & 0.1453 & 0.1370 & 0.1452 & 0.1370 \\
\hline 2 & $0.1514(0.1504)$ & 0.1384 & 0.1515 & 0.1385 & 0.1515 & 0.1385 & 0.1515 & 0.1386 \\
\hline 3 & $0.1282(0.1267)$ & 0.1352 & 0.1281 & 0.1351 & 0.1281 & 0.1350 & 0.1281 & 0.1350 \\
\hline 4 & $0.1400(0.1417)$ & 0.1320 & 0.1400 & 0.1321 & 0.1400 & 0.1322 & 0.1400 & 0.1322 \\
\hline 5 & $0.1388(0.1367)$ & 0.1496 & 0.1388 & 0.1496 & 0.1388 & 0.1497 & 0.1388 & 0.1497 \\
\hline 6 & $0.1361(0.1362)$ & 0.1242 & 0.1360 & 0.1241 & 0.1360 & 0.1241 & 0.1359 & 0.1241 \\
\hline 7 & $0.1460(0.1454)$ & - & 0.1461 & - & 0.1462 & - & 0.1462 & - \\
\hline mean & $0.1400(0.1401)$ & 0.1361 & 0.1400 & 0.1361 & 0.1400 & 0.1361 & 0.1399 & 0.1361 \\
\hline BLA $^{14}$ & - & - & 0.0077 & 0.0076 & 0.0064 & 0.0064 & 0.0060 & 0.0059 \\
\hline
\end{tabular}




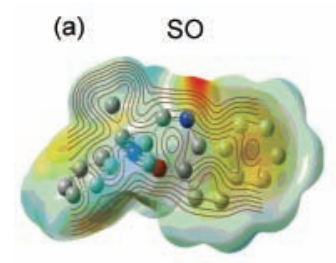

SO1

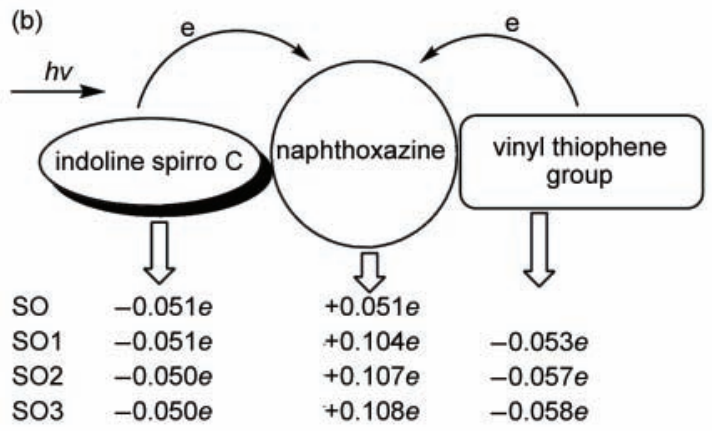

图 2 (a) SO 和 $\mathrm{SO} 1$ 的电荷密度剖面图以及(b) SO-SO3 的 基态和第一单激发态的电荷分布

Fig. 2 (a) Profile diagram of charge density for SO and SO1 and (b) charge distribution on the ground and first single state of $\mathrm{SO}-\mathrm{SO} 3$

分子在由基态到激发态过程中, 含螺 $\mathrm{C}$ 吲哚环部分 和乙烯基噻吩单元部分分别有大小不等的电子 流向菜并惡嗪部分, 其中, 比较 $\mathrm{SO}$ 和 $\mathrm{SO} 1$ 的荎并 噁嗪部分的电荷, 发现后者 $(+0.104 e)$ 几乎是前者 $(+0.051 e)$ 的两倍, 随着乙烯基噻吩单元的增加, 电荷 变化逐渐变大, 说明乙烯基噻吩基团可以显著增强 螺噁嗪分子的萗并噁嗪部分的电子流动.

\section{3 前线分子轨道}

HOMO 和 LUMO 对分子性质的影响非常重 要. ${ }^{15,16}$ 图 3 和图 4 分别给出了 B3LYP/6-31G*计算所 得 $\mathrm{SO}-\mathrm{SO} 3$ 及其对应开环体的前线分子轨道, 即 HOMO 和 LUMO 的轨道及能量, 图 5 给出了其各个 部分与整体态密度分布状况, 图 6 给出了 $\mathrm{SO}-\mathrm{SO} 3$ 以及其开环体的能隙变化.

根据前线轨道理论, 螺噁嗪的分子轨道可以由 吲哚环和菜并啞嗪两部分相互作用构成. 由图 3 和 图 5 可见, $\mathrm{SO}$ 的 $\mathrm{HOMO}$ 主要分布在吲哚环(占 77\%) 而 LUMO 主要分布在菜并噁嗪部分(占 $96 \%$ ), 在 SO 中, 由于轨道分布在由螺碳原子连接的两个不同 的部分因此并没有发生明显的作用, 其 HOMOLUMO 的跃迁主要对应于从吲哚环到菜并噁嗪的 电荷转移跃迁, 基于前线轨道对称性, 这种跃迁的 强度很低. 当萗环上连接不同长度的乙烯基噻吩基 团后, $\mathrm{SO} 1-\mathrm{SO} 3$ 分子的前线轨道较 $\mathrm{SO}$ 发生变化即 $\mathrm{HOMO}$ 和 LUMO 基本都分布在除吲哚环外的 $\pi$ 共轭 骨架上(由荎并噁嗪与乙烯基噻吩两部分组成), 并
分别显示出典型的 $\pi$ 和 $\pi^{*}$ 轨道特征. 乙烯基噻吩单元 在 HOMO 和 LUMO 中的轨道贡献率分别由 $45 \%$ 和 $31 \%$ 增加到 $86 \%$ 和 $90 \%$, 这说明由于乙烯基噻吩本 身强的 $\pi$ 电子的离域性, 改变了螺噁嗪母体电子云 分布以及前线分子轨道的分布.

同时, 能隙在逐渐减小, 说明乙烯基噻吩的 引入增加了其与荟并噁嗪部分的共轭作用; 其中, HOMO 能均增大, 体系易失去电子, 即易产生空穴; LUMO 能均减小, 表明体系易接受电子, 电子注入 能力提高了, 因此使得电子云流动变得更加容易. 由图 6 可以发现, 开环体的能隙较未开环的明显减 小, 这主要是开环过程分子结构形成了一个更大的 $\pi$ 共轭体系造成的(由图 4 的 HOMO 轨道分布可见).

\section{4 吸收光谱}

用含时密度泛函 TD-B3LYP/6-31G*计算得到

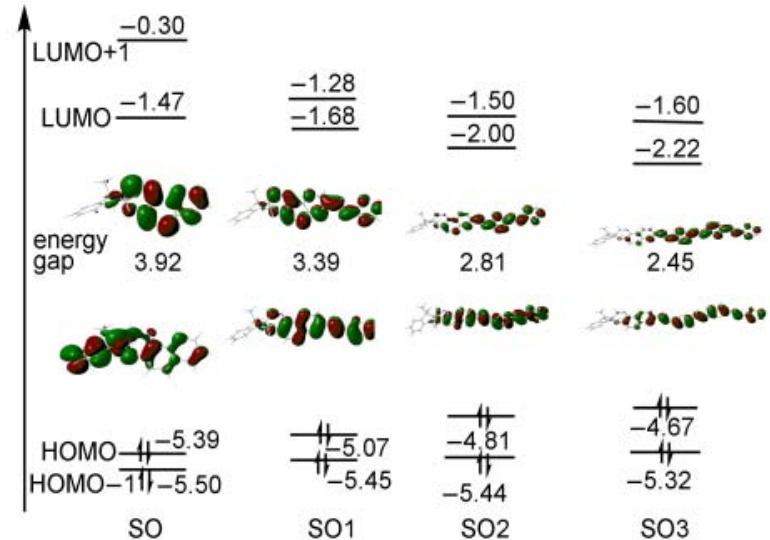

图 $3 \mathrm{~B} 3 \mathrm{LYP} / 6-31 \mathrm{G} *$ 计算所得 SO-SO3 的前线分子 轨道及能隙 $(\mathrm{eV})$

Fig.3 Frontier molecular orbitals and energy gap (eV) of SO-SO3 calculated using B3LYP/6-31G*

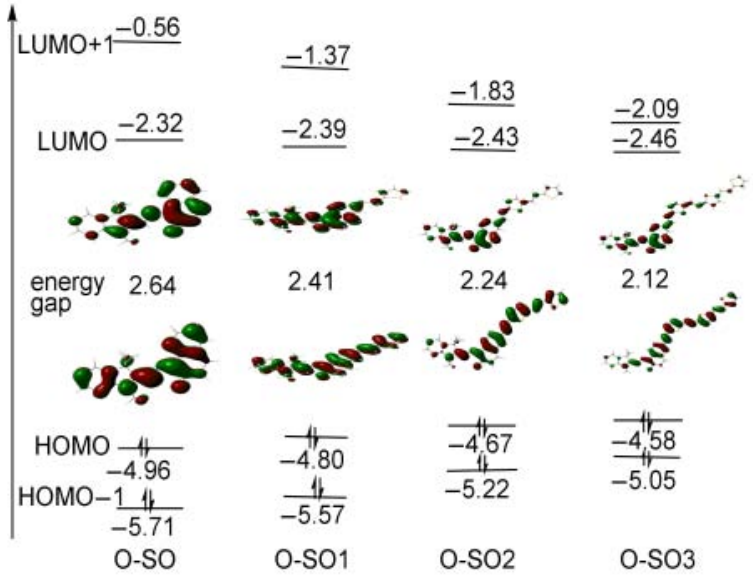

图 $4 \mathrm{~B} 3 \mathrm{LYP} / 6-31 \mathrm{G}$ *计算所得 SO-SO3 对应的开环体的 前线分子轨道及能隙 $(\mathrm{eV})$

Fig.4 Frontier molecular orbitals and energy gap (eV) of the corresponding open-forms of $\mathrm{SO}-\mathrm{SO} 3$ calculated using B3LYP/6-31G* 

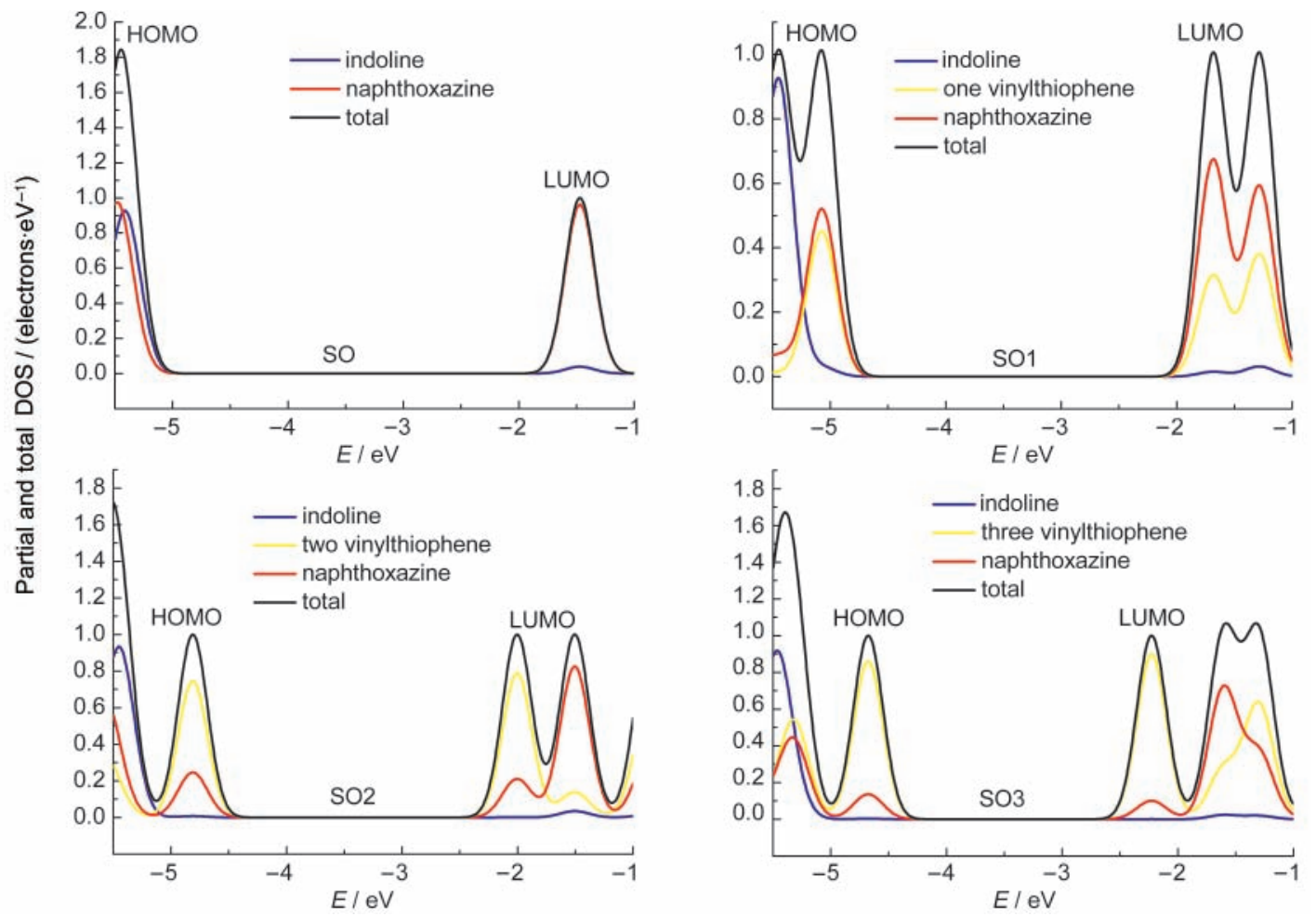

图 $5 \mathrm{SO}-\mathrm{SO3}$ 的各个部分与整体态密度(DOS)分布图

Fig.5 Diagrams of partial and total density of states (DOS) for SO-SO3

$\mathrm{SO}-\mathrm{SO} 3$ 及其开环体的电子吸收光谱, 最低三个单 重激发态 $\left(S_{1}-S_{3}\right)$ 及其对应的吸收波长 $\lambda$, 振子强度 $f$ 以及跃迁类型. 由表 2 可见, 对于 $\mathrm{SO}$ 分子, TDDFT 的计算预测与文献 ${ }^{10(\mathrm{c}, \mathrm{d})}$ 实验值显示了良好的的一致 性, 说明了此计算方法的可行性. 为了讨论不同基 组下对于 TDDFT 计算电子光谱的影响, 分别在 6-31G*, 6-31+G*, 以及 6-311+G*下对 $\mathrm{SO}$ 分子进行 了 TD-DFT 的电子光谱计算, 由表 2 可以发现基组 增加弥散函数后, 吸收波长变化不大, 相对于基组 6-31G*仅发生 4-6 nm 的红移, 说明对于本体系在 TD-B3LYP/6-31G*下达到计算精度和计算效率的最

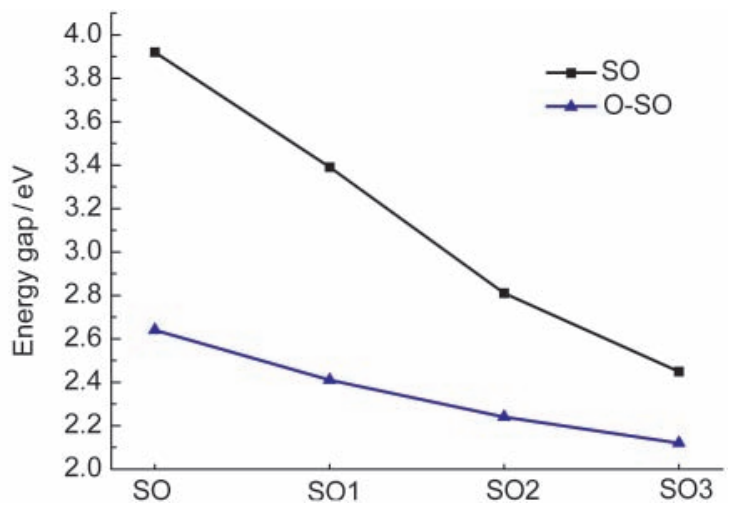

图 $6 \mathrm{SO}-\mathrm{SO3}$ 及其对应开环体的能隙

Fig.6 Energy gap of SO-SO3 and the corresponding open-forms
优化. 但计算所得结果与实验结果略有偏差, 造成 计算结果与实验值偏差的原因是计算方法和实验 方法都有各自的缺陷, 比如计算中都需要引入一些 近似, 计算值所处的环境是在真空态下, 但因理论 计算结果中考虑了必要的相关效应, 保留了重要因 素的作用, 对于实验预测仍是可信的.

文献 ${ }^{17}$ 报道螺噁嗪类化合物开环体的形成只经 过激发单重态形成的. ${ }^{18}$ 由表 2 可知, SO 的 HOMO 和 LUMO 分布在不同的环上, 因此其电子跃迁相对 具有较弱的振子强度 $(f=0.044)$, 同时影响其最大吸 收波长的最低能量激发态发生在第二单重态 $S_{2}$. 而 $\mathrm{SO} 2$ 和 $\mathrm{SO} 3$ 的最大吸收波长发生在最低单重激发态 $S_{1}, S_{1}$ 均源自电子从 HOMO-LUMO 的跃迁且为 $\pi-\pi^{*}$ 跃迁, 并且分别具有较强的振子强度 $(f=1.910$ 和 $f=$ 2.758), 这说明随着乙烯基噻吩单元的增加, 开环所 需激发能降低, 将更容易发生激发开环.

由图 7 可见, 计算所得 SO-SO3 的最大吸收波 长分别为 $344 、 364 、 466 、 540 \mathrm{~nm}$, 可见由于乙烯基噻 吩与螺噁嗪慕环部分的共轭作用, 使得整体的吸收 发生了显著的红移, 特别是摩尔消光系数 $\varepsilon$ 由 $10^{3}$ 增 大到 $10^{5}$, 在此位置的吸收强度明显增大. 值得注意 的是 $\mathrm{SO}-\mathrm{SO} 3$ 的最大吸收波长由紫外激发区转移 到可见光激发区. 此外, 在同样的计算方法和基组 
表 2 TD-B3LYP 计算所得SO-SO3 的电子吸收光谱, 最低三个单重激发态及其对应的吸收波长 $\lambda 、$ 振子强度 $f$ 以及跃迁类型

Table 2 Electronic absorption spectra, wavelengths $\lambda$, oscillator strengths $f$, and the lowest three singlet excited states of SO-SO3 calculated with TD-B3LYP

\begin{tabular}{ccccl}
\hline Compound & Transition & $\lambda / \mathrm{nm}$ & $f$ & \multicolumn{1}{c}{ Description } \\
\hline SO & $S_{0}-S_{1}$ & $374^{\mathrm{a}} 376^{\mathrm{b}} 377^{\mathrm{c}} 369(\mathrm{expt})^{\mathrm{d}}$ & 0.044 & $\mathrm{H} \rightarrow \mathrm{L}(89 \%)^{\mathrm{c}}$ \\
& $S_{0}-S_{2}$ & $344^{\mathrm{a}} 349^{\mathrm{b}} 351^{\mathrm{c}} 345(\mathrm{expt})^{\mathrm{d}}$ & 0.135 & $\mathrm{H}-1 \rightarrow \mathrm{L}(79 \%)$ \\
& $S_{0}-S_{3}$ & $301^{\mathrm{a}} 304^{\mathrm{b}} 306^{\mathrm{c}} 317\left(\operatorname{expt}^{\mathrm{d}}\right.$ & 0.044 & $\mathrm{H}-2 \rightarrow \mathrm{L}(66 \%)$ \\
$\mathrm{SO} 1$ & $S_{0}-S_{1}$ & 401 & 0.228 & $\mathrm{H} \rightarrow \mathrm{L}(69 \%)$ \\
& $S_{0}-S_{2}$ & 364 & 0.702 & $\mathrm{H}-1 \rightarrow \mathrm{L}(78 \%)$ \\
& $S_{0}-S_{3}$ & 337 & 0.021 & $\mathrm{H} \rightarrow \mathrm{L}+1(67 \%)$ \\
$\mathrm{SO} 2$ & $S_{0}-S_{1}$ & 467 & 1.910 & $\mathrm{H} \rightarrow \mathrm{L}(86 \%)$ \\
& $S_{0}-S_{2}$ & 421 & 0.093 & $\mathrm{H} \rightarrow \mathrm{L}+1(85 \%)$ \\
& $S_{0}-S_{3}$ & 394 & 0.002 & $\mathrm{H}-1 \rightarrow \mathrm{L}(84 \%)$ \\
& $S_{0}-S_{1}$ & 540 & 2.758 & $\mathrm{H} \rightarrow \mathrm{L}(78 \%)$ \\
& $S_{0}-S_{2}$ & 455 & 0.001 & $\mathrm{H} \rightarrow \mathrm{L}+1(54 \%)$ \\
& $S_{0}-S_{3}$ & 426 & 0.051 & $\mathrm{H}-1 \rightarrow \mathrm{L}(30 \%)$ \\
\hline
\end{tabular}

${ }^{a}$ calculated with TD-B3LYP/6-31G*. ${ }^{b}$ calculated with TD-B3LYP/6-31+G*. ${ }^{c}$ calculated with TD-B3LYP/6-311+G*. ${ }^{\text {d}}$ Ref.17.

${ }^{\circ} \mathrm{H}$ and $\mathrm{L}$ represent $\mathrm{HOMO}$ and LUMO, respectively, the percentage contributions to wave functions of excited states are given in parentheses.

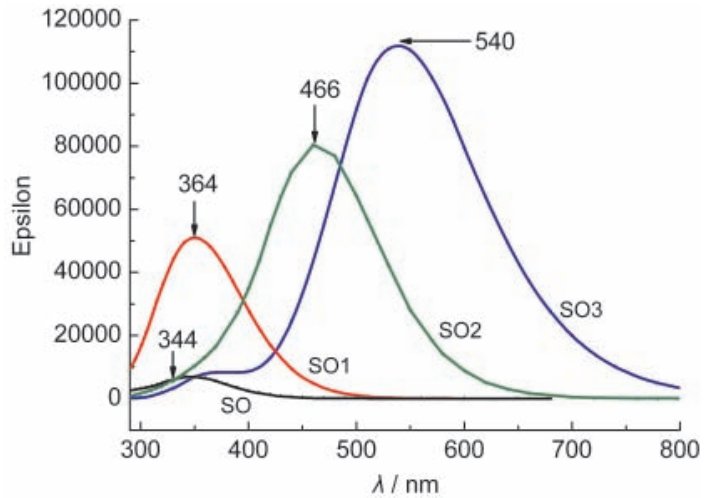

图 7 TD-B3LYP/6-31G*计算所得的 SO-SO3 的 电子吸收光谱

Fig.7 Electronic absorption spectra of $\mathrm{SO}-\mathrm{SO} 3$ calculated with TD-B3LYP/6-31G*

下计算研究 SO-SO3 对应的开环体的电子吸收光 谱(表 3), 对于 O-SO 而言, 其最低单重激发态 $S_{1}$ 为 $\sigma \rightarrow \pi^{*}(\mathrm{LUMO})$ 跃迁, 其中 $\sigma$ 轨道主要来源于氧原子和 氮原子上的孤对电子并分布在噁嗪环上, 根据轨道 对称性, 这种跃迁是禁阻的且其振子强度几乎可以 忽略. 而影响 O-SO 最大吸收的 $S_{0}-S_{2}$ 跃迁表现为 $\pi-\pi^{*}$ 特征, 且主要来源于电子从 HOMO 到 LUMO 的 激发跃迁, 由图 4 可见其 HOMO 和 LUMO 的轨道均 分布在整个共轭平面结构上. 对于 $\mathrm{O}-\mathrm{SO} 2$ 和 $\mathrm{O}-\mathrm{SO} 3$, 其最大吸收波长发生在最低单重激发态 $S_{1}$, 均源自电子从 HOMO 到 LUMO 的跃迁且同样呈现 为典型 $\pi-\pi^{*}$ 跃迁. 此外, 从表 3 可以发现开环体吸收 波长的变化趋势与闭环体类似, 随着乙烯基噻吩单 元的增加, 从 $\mathrm{O}-\mathrm{SO}$ 到 $\mathrm{O}-\mathrm{SO} 3$ 其吸收波长发生约 $68-170 \mathrm{~nm}$ 的红移, 其中 $\mathrm{O}-\mathrm{SO} 2$ 与 $\mathrm{O}-\mathrm{SO} 3$ 的最大吸 收波长达到 605 和 $647 \mathrm{~nm}$, 相应的开环体理论上会 在可见光区产生不同的颜色. 由于接入 2-3 个乙烯

表 3 TD-B3LYP/6-31G*计算所得 SO-SO3 对应的开环体的电子吸收光谱, 最低三个单重激发态及其对应的吸收波长 $\lambda 、$ 振子强度 $f$ 以及跃迁类型

Table 3 Electronic absorption spectra, wavelengths $\lambda$, oscillator strengths $f$, the lowest three singlet excited states of the corresponding open-forms of SO-SO3 calculated with TD-B3LYP/6-31G*

\begin{tabular}{cccll}
\hline Compound & Transition & $\lambda / \mathrm{nm}$ & \multicolumn{1}{c}{ Description } \\
\hline O-SO & $S_{0}-S_{1}$ & 554 & 0.000 & $\mathrm{H}-1 \rightarrow \mathrm{L}(93 \%)$ \\
& $S_{0}-S_{2}$ & 476 & 0.729 & $\mathrm{H} \rightarrow \mathrm{L}(70 \%)$ \\
& $S_{0}-S_{3}$ & 402 & 0.065 & $\mathrm{H}-2 \rightarrow \mathrm{L}(89 \%)$ \\
O-SO1 & $S_{0}-S_{1}$ & 551 & 0.228 & $\mathrm{H} \rightarrow \mathrm{L}(69 \%)$ \\
& $S_{0}-S_{2}$ & 544 & 0.801 & $\mathrm{H} \rightarrow \mathrm{L}(78 \%)$ \\
O-SO2 & $S_{0}-S_{3}$ & 432 & 0.454 & $\mathrm{H}-1 \rightarrow \mathrm{L}(67 \%)$ \\
& $S_{0}-S_{1}$ & 605 & 0.943 & $\mathrm{H} \rightarrow \mathrm{L}(80 \%)$ \\
& $S_{0}-S_{2}$ & 556 & 0.000 & $\mathrm{H} \rightarrow \mathrm{L}+1(85 \%)$ \\
O-SO3 & $S_{0}-S_{3}$ & 494 & 0.924 & $\mathrm{H}-1 \rightarrow \mathrm{L}(84 \%)$ \\
& $S_{0}-S_{1}$ & 647 & 1.311 & $\mathrm{H} \rightarrow \mathrm{L}(86 \%)$ \\
& $S_{0}-S_{2}$ & 556 & 0.001 & $\mathrm{H}-3 \rightarrow \mathrm{L}(83 \%)$ \\
& $S_{0}-S_{3}$ & 549 & 1.305 & $\mathrm{H} \rightarrow \mathrm{L}+1(67 \%)$ \\
\hline
\end{tabular}


基噻吩单元使得能够激发闭环体开环的最大吸收 波长转移到可见光区, 同时相应开环体在可见光区 呈现出颜色, 这就为设计和合成新型可见光致变色 材料提供了可靠的理论依据, 并且将会极大地拓展 这类光学材料的应用范围.

\section{4 结 论}

采用密度泛函理论和含时密度泛函方法, 分别 在 B3LYP/6-31G*水平下研究和预测了乙烯基噻吩 共轭螺噁嗪衍生物的几何构型、电子结构和电子吸 收光谱并得到了较为理想的结果: (1) 理论计算预测 的结果和文献已有的实验值吻合较好, 说明了理论 计算的可靠性. (2) SO-SO3 的开环过程会使得其共 轭体系变大, 带隙明显减小, 最大吸收波长红移. (3) 乙烯基噻吩基团共轭接入吲哚啉螺萗并噁嗪母体, 由于乙烯基噻吩本身强的 $\pi$ 电子的离域性, 使得乙 烯基噻吩与菜并噁嗪部分共轭作用增大; 改变了菜 并噁嗪部分的电子云分布同时使电子流动更容 易, 增加了整个分子的极性, 降低了分子的带隙, 改变了前线分子轨道分布特别是乙烯基噻吩单元 在 HOMO 和 LUMO 中的轨道贡献率明显增加; 当 接入的乙烯基噻吩单元达到 2-3 个时, $\mathrm{SO} 2$ 和 $\mathrm{SO} 3$ 的最低激发单重态 $\left(S_{1}\right)$ 均源自电子从 HOMO 到 LUMO 的跃迁, 且为 $\pi-\pi^{*}$ 跃迁; 影响最大吸收波长 的最低能量激发态变为 $S_{1}$, 激发开环将变得容易. 最 大吸收波长 $\lambda_{\text {max }}$ 达 466-540 nm, 红移十分明显并出 现在可见光区, 对光子的吸收效率显著增加, 使得 激发光源从紫外光向可见光区域发生转变, 其中 $\mathrm{O}-\mathrm{SO} 2$ 与 $\mathrm{O}-\mathrm{SO} 3$ 的最大吸收波长达到 605 和 647 $\mathrm{nm}$, 开环体会在可见光区产生相应的颜色变化(如 从绿色到蓝色). 这为设计和合成新型可见光致变色 及光电转化材料提供了可靠的理论依据, 将会极大 地拓展这类光学材料的应用范围. 此外, 了解化学 结构和电子吸收光谱等性质是对具有优良性能的 材料进行分子结构设计和指导实验合成的起点, 在 DFT 的理论计算框架下尤其是含时密度泛函的应 用进行研究显示了与实验结果的规律的一致性, 将 为今后改构设计分子提供可靠的参考依据. ${ }^{19-21}$

\section{References}

(1) Fox, R. E. Research Reports and Test Items Pertaining to Eye Protection of Air Crew Personnel. USA Patent AD440226 [P]. 1961 Final Report on Contract AF41 (657) 215, AD 440226, 1961.
(2) Fan, M. G. Basic Fundamentals of Photochemistry and Materials Science of Photonics; Science Press: Beijing, 2001; p 226. [樊美公. 光化学基本原理与光子学材料科学. 北京: 科学出版社, 2001: 226.]

(3) Tamai, N.; Miyasaka, H. Chem. Rev. 2000, 100, 1875.

(4) Chu, N. Y. C. Can. J. Chem. 1983, 61, 300.

(5) Tang, Z. Y.; Hu, Y. C.; Zhao, Y.; Liu, S. B. Acta Phys. -Chim. Sin. 2009, 25, 701. [唐智勇, 胡云楚, 赵 莹, 刘述斌. 物理 化学学报, 2009, 25, 701.]

(6) Xue, Y. S.; Gong, X. D.; Xiao, H. M.; Tian, H. Acta Chim. Sin. 2004, 62, 963. [薛运生, 贡雪东, 肖鹤鸣, 田 禾. 化学学报, 2004, 62, 963.]

(7) Wang, J. Y.; Feng, C. G. Chinese Journal of Applied Chemistry 2007, 24, 7. [王建营, 冯长根. 应用化学, 2007, 24, 7.]

(8) Kanis, D. R.; Ratner, M. A.; Marks, T. J. Chem. Rev. 1994, 94, 195.

(9) Dehu, C.; Meyers, F.; Bredas, J. L. J. Am. Chem. Soc. 1993, 115, 6198.

(10) (a) Lee, C.; Yang, W.; Parr, R. G. Phys. Rev. B 1988, 37, 785. (b) Becke, A. D. J. Chem. Phys. 1993, 98, 5648. (c) Maurel, F.; Aubard, J.; Millie, P.; Dognon, J. P.; Rajzmann, M.; Guglielmetti, R.; Samat, A. J. Phys. Chem. A 2006, 110, 4759 .

(d) Sheng, Y. H.; Leszczynski, J.; Garcia, A. A.; Rosario, R.; Gust, D.; Springer, J. J. Phys. Chem. B 2004, 108, 16233.

(11) Frisch, M. J.; Trucks, G. W.; Schlegel, H. B.; et al. Gaussian 09, Revision B.01; Gaussian Inc.: Pittsburgh, PA, 2010.

(12) (a) Schneider, S. Z. Phys. Chem. Neue. Folge. 1987, 154, 91. (b) Maeda, S.; Mitsuhashi, K.; Osano, Y. T.; Nakamura, S.; Ito, M. Mol. Cryst. Liq. Cryst. 1994, 246, 223.

(13) Millini, R.; Del Piero, G.; Allegrini, P.; Crisci, L.; Malatesta, V. Acta Crystallogr. C 1991, 47, 2567.

(14) Ortiz, R. P.; Delgado, M. C. R.; Casado, J.; Hernández, V.; Kim, O.; Woo, H. Y.; Navarrete, J. T. L. J. Am. Chem. Soc. 2004, 126, 13363.

(15) Cufioni, A.; Andreoni, W. IBM J. Res. Dev. 2001, 45, 101.

(16) Cufioni, A.; Mauro, B.; Andreoni, W. Chemical Physics Letters 1998, 294, 263.

(17) Bohne, C.; Fan, M. G.; Li, Z. J.; Lusztyk, J.; Scaiano, J. C. J. Chem. Soc. Chem. Commun. 1990, 571.

(18) (a) Tyer, N. W., Jr.; Becker, R. S. J. Am. Chem. Soc. 1970, 92, 1289.

(b) Tyer, N. W., Jr.; Becker, R. S. J. Am. Chem. Soc. 1970, 92 , 1295 .

(19) Wang, X. W.; Jiang, G.; Du, J. G. Acta Phys. -Chim. Sin. 2011, 27, 309. [王晓巍, 蒋 刚, 杜际广. 物理化学学报, 2011, 27, 309.]

(20) Kumar, S.; Velasco, K.; McCurdy, A. J. Mol. Struct. 2010, 968, 13.

(21) Hudson, G. A.; Cheng, L.; Yu, J. M.; Yan, Y.; Dyer, D. J.; McCarroll, M. E.; Wang, L. C. J. Phys. Chem. B 2010, 114, 870. 